\title{
e-Phaïstos
}

e-Phaïstos

Revue d'histoire des techniques / Journal of the history

of technology

IX-1 | 2021

Autour de Léonard de Vinci

\section{Léonard entre deux mondes}

Culture des bibliothèques et culture des ateliers en confrontation au temps de la révolution scientifique

Leonardo as a Go-Between: Bookshop's Culture versus Workshop's Culture during the Scientific Revolution

\section{Laurent-Henri Vignaud}

\section{OpenEdition}

\section{Journals}

Édition électronique

URL : https://journals.openedition.org/ephaistos/8766

DOI : $10.4000 /$ ephaistos.8766

ISSN : 2552-0741

Éditeur

IHMC - Institut d'histoire moderne et contemporaine (UMR 8066)

Référence électronique

Laurent-Henri Vignaud, "Léonard entre deux mondes », e-Phaïstos [En ligne], IX-1 | 2021, mis en ligne le 30 avril 2021, consulté le 20 septembre 2021. URL : http://journals.openedition.org/ephaistos/8766 ; DOI : https://doi.org/10.4000/ephaistos.8766

Ce document a été généré automatiquement le 20 septembre 2021.

Tous droits réservés 


\title{
Léonard entre deux mondes
}

\author{
Culture des bibliothèques et culture des ateliers en confrontation au \\ temps de la révolution scientifique
}

Leonardo as a Go-Between: Bookshop's Culture versus Workshop's Culture during the Scientific Revolution

Laurent-Henri Vignaud

1 Léonard partage sans doute avec Galilée ce rare mais désagréable privilège d'avoir suscité des appréciations extrêmement contradictoires (Stengers 2003). Le jugement porté sur cet «homme universel» (le mot est de Paul Valéry) n'est pas simplement affaire d'images d'épinal ${ }^{1}$, il a son écho historiographique. Le mythe, encore très populaire de nos jours, du «miracle » Léonard, génie fulgurant qui sait tout et imagine tout avant les autres, n'a cependant plus cours parmi les spécialistes. S'il y a une «énigme Léonard ", elle se résout dans l'étude du milieu des ingénieurs italiens et des artisans florentins de la Renaissance auprès desquels il s'est formé. C'est en tout cas le point de vue presque unanimement partagé par les historiens actuels (Vecce 2001, Brioist 2019).

2 La sanctification de Léonard a commencé au lendemain de sa mort. Vasari, qui, il est vrai, modère rarement ses éloges, ne dit-il pas que sa vie témoigne «moins de la puissance du génie humain que de la faveur spéciale de Dieu $^{2}$ » ? Il s'agit pourtant ici de glorifier, sur un ton très platonicien, les talents de peintre, de sculpteur et d'architecte de l'artiste. Le Léonard grand savant et ingénieur surgit bien plus tard avec la découverte et la publication des manuscrits (Atlanticus, Arundel, Leicester, Madrid, etc.), à une époque charnière de l'histoire occidentale du point de vue de l'évolution des techniques: la fin du XIX et le début du $\mathrm{XX}^{\mathrm{e}}$ siècle, au moment où apparaît une véritable «culture de l'innovation » (Garçon 2017). En plein triomphe du machinisme, la mise au jour de ces incroyables dessins anticipant la réalisation d'avions, de bicyclettes, de chars d'assaut et d'aérostats contribue à faire du peintre florentin « un phare qui éclaire de ses rayons jusqu'aux horizons des âges à venir, les premiers pas de l'art et de la science modernes ${ }^{3} »$ (Ronna $1902: 129$ ). 
3 A contrario, il est cet homme "sans lettres ", de son propre aveu, qu'on aurait du mal à comparer à certains de ses contemporains, mieux versés que lui dans la culture humaniste. Tel est le cas de Taccola, surnommé l'Archimède de Sienne, ou d'Alberti, auquel on l'a souvent opposé, à son détriment. Sa langue est celle d'un fermier toscan, son écriture est phonétique, et s'il lui arrive de rédiger ses notes à l'envers, ce n'est pas pour tenir secrètes des découvertes inouïes ni pour préserver ses petites astuces à la manière d'un artisan. D'un autre côté, il n'est pas non plus l'ignare chef d'atelier que d'autres ont voulu décrire puisqu'il possède une honnête bibliothèque d'environ 200 livres et qu'il acquiert avec l'âge un goût de plus en plus prononcé pour la théorie et la modélisation. Paolo Rossi suggère que c'est ce double visage de Léonard qui explique qu'il soit devenu, dès son époque, le «symbole du dépassement de cette mentalité qui opposait radicalement arts libéraux et arts mécaniques » (Rossi 1996 : 28).

Or, précisément, ce dépassement ne s'est pas fait en un jour ni même en une vie, fûtelle celle d'un "génie ». L'opposition classique (aristotélicienne puis scolastique) entre épistémè et technè perdure au-delà de la Renaissance et elle pose problème à chacune des grandes étapes du développement des sciences et des techniques occidentales jusqu'à la révolution industrielle (Garçon 2007).

5 Les remarques qui suivent et qui portent sur des exemples empruntés au XVII ${ }^{e}$ siècle visent à montrer la persistante opposition de vues entre savants, ingénieurs et artisans maintenue jusqu'à l'émergence, au cours du XVIII ${ }^{e}$ siècle, d'une technoscience. Le dialogue entre savants et techniciens, appelés à collaborer étroitement à partir de la Renaissance, confine parfois à un dialogue de sourds. Pourtant, les savants ont de plus en plus fréquemment recours aux techniciens et aux artisans pour fabriquer les machines avec lesquelles ils procèdent à des démonstrations expérimentales. Les liens étroits entre Boyle et son préparateur Hooke dans la conception puis la manipulation de la fameuse " pompe à air » en sont devenus le paradigme historiographique (Shapin 1998). Peut-être un peu plus tardivement, les ingénieurs et artisans font à leur tour usage des savoirs théoriques pour construire leurs machines, améliorer leurs procédés ou réaliser leurs plans. Le monde "technologique» dans lequel nous évoluons aujourd'hui est l'aboutissement de ce rapprochement. Mais ce processus de fusion, qui n'est ni immédiat ni total, se heurte à plusieurs reprises dans l'histoire des sciences et des techniques à de grands moments d'incompréhension, non sans rapport avec les diverses appréciations du personnage Léonard apparues au fil du temps. Qu'on le veuille ou non, les deux cultures, scientifique et technique, ne sont pas entièrement commensurables.

\section{Descartes et la fabrication de lentilles hyperboliques}

Dès l'invention de la lunette astronomique, avec les polémiques qu'elle engendre entre les partisans de Galilée et ses adversaires, les astronomes qui utilisent ce nouvel instrument sont gênés par un effet indésirable des lentilles appelé aberration chromatique. Les capacités de dispersion du verre interdisent que la mise au point puisse être effectuée simultanément pour toutes les couleurs du spectre : certaines couleurs apparaissent claires et d'autres floues, produisant des phénomènes d'irisation très incommodes pour l'observation des cieux. Les astronomes n'ont alors de cesse de résoudre ce problème, finalement surmonté par l'invention des lentilles achromatiques (fabriquées en flint-glass, un verre comprenant à l'origine de l'oxyde de plomb) par un 
opticien anglais vers 1730, puis breveté en 1758 par le facteur d'instruments londonien John Dollond. Avant cette date, diverses solutions théoriques et pratiques sont imaginées pour empêcher l'aberration. L'une d'entre elles est élaborée par Descartes. Il en livre le secret, dessins à l'appui, dans le dixième discours de sa Dioptrique (1637) ${ }^{4}$. Les illustrations contenues dans cet ouvrage sont celles d'une machine à polir les lentilles de son invention capable de tailler les verres en forme hyperbolique, laquelle forme est censée, selon les lois de l'optique que Descartes a lui-même établies, supprimer le défaut chromatique ${ }^{5}$. Cette machine ne fonctionna pourtant jamais, mais ce ne fut pas faute d'essayer (Michaux 2001).

7 Les vicissitudes conduisant à l'abandon de ce projet de machine comme à son intégration au dernier discours de la Dioptrique nous sont connues par la correspondance de Descartes (Burnett 2005). Entre juin 1629 et décembre 1638, soit durant près de dix ans, Descartes s'entretient avec un artisan parisien nommé Ferrier de la fabrication de sa polisseuse de lentilles hyperboliques. Les relations entre les deux hommes sont, au début, excellentes et faites de respect mutuel. L'échec du projet n'est donc pas dû à des incompatibilités d'humeurs ou à une quelconque brouille. Simplement, Ferrier ne parvient à répondre aux exigences du savant et celui-ci ne saisit pas l'enjeu des difficultés matérielles auxquelles se heurte le premier. Au fil des essais, Ferrier informe Descartes, dans la plus complète indifférence du savant, des difficultés techniques qu'il rencontre: il mentionne des problèmes de résistance des matériaux, d'usure précoce des pièces, de vibrations qui fausse la taille, de manque de précision, etc. Ainsi, dans une longue lettre adressée par Ferrier à Descartes après ses premiers essais, l'artisan évoque la contrainte, purement efficiente, d'évacuation des résidus de taille :

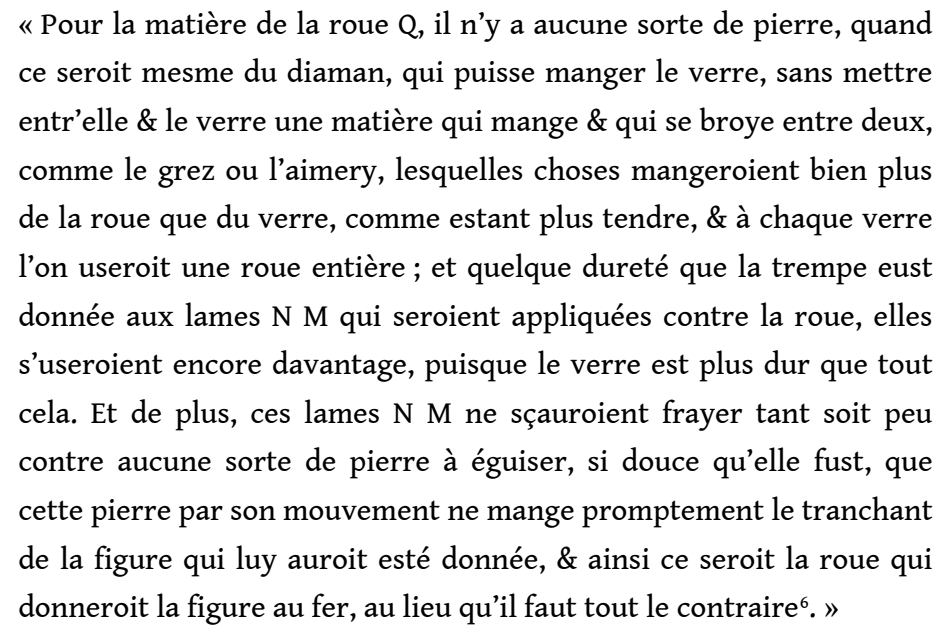

Pour Descartes, ces remarques n'ont guère de sens : il raisonne en général d'armée pour qui l'intendance doit suivre quoi qu'il arrive... L'objet hyperbolique est un objet parfait du point de vue mathématique; il a la forme idéalement adaptée pour résoudre les irisations. Sa constructibilité est dès lors supposée acquise. Si l'artisan échoue, c'est qu'il n'aura pas su s'y prendre ${ }^{7}$. Rien ne fait dévier Descartes de cette pensée, même lorsque Constantin Huygens lui conseille en 1635 un "tourneur d'Amsterdam » en remplacement de Ferrier. Les mêmes ennuis ne tardent pas à surgir et la machine hollandaise à polir les lentilles hyperboliques n'est pas plus construite que la parisienne. 
9 À la fin de la Dioptrique, le philosophe et mathématicien fait le constat, un peu amer, de son échec à faire fabriquer ladite machine (qu'il impute à la défaillance des artisans) mais se félicite d'en avoir eu l'idée :

\begin{abstract}
"Je ne vous parle point de plusieurs autres particularités qu'on doit observer en les taillant, ny aussi de plusieurs autres choses que j'ay tantost dit estre requises en la construction des lunetes, car il n'y en a aucune que je juge si difficile qu'elle puisse arrester les bons esprits, et je ne me reigle pas sur la portée ordinaire des artisans, mais je veux esperer que les inventions que j'ay mises en ce Traité seront estimées assés belles et assés importantes pour obliger quelques-uns des plus curieux et des plus industrieux de nostre siecle à en entreprendre l'exécution ${ }^{8}$.»
\end{abstract}

10 Comme à son habitude, Descartes parie sur un avenir proche pour venir à bout du problème. En cela, il ne s'éloigne pas tant d'une certaine forme d'ingenium propre à Léonard qui, ainsi que le souligne Hélène Vérin, se livre à une "surenchère inventive de machines et agencements souvent impraticables» (Vérin 1993 : 30). Il n'accomplit cependant pas une réflexion technique consistant à améliorer les mécanismes de sa machine afin de répondre aux difficultés rencontrées par Ferrier. Tous ses efforts portent sur la constructibilité mathématique de l'engin, pas sur sa constructibilité mécanique et matérielle. Prudent néanmoins, Descartes n'entendait payer à Ferrier que les pièces réussies, exigence qu'il croyait suffisante pour vaincre tous les obstacles et tester la bonne volonté de l'artisan. Le plus étonnant est qu'au bout du compte Descartes finit par ne pas prendre au sérieux cette bonne volonté quand Ferrier énonce en toute bonne foi les singularités de la constructibilité technique.

\title{
Pascal et la pascaline
}

11 Un autre épisode bien connu de l'incompréhension mutuelle entre savants et artisans au XVII ${ }^{\mathrm{e}}$ siècle concerne la machine à calculer de Pascal. On connaît les circonstances de son invention: fils d'un agent du fisc royal, Pascal entend soulager son père des nombreuses opérations de calcul que celui-ci effectue au quotidien. La conception de la machine est une véritable prouesse: elle additionne et soustrait, par combinaison multiplie et divise, le tout en base 10 ou 12 (correspondant à la monnaie de compte de l'époque). Un "reporteur à sautoir ", invention de Pascal, effectue automatiquement les retenues. Cette pièce technique suscite l'admiration des savants: Bellair et Huygens en discutent dans leur correspondance, un dessin précis est inséré dans l'Encyclopédie de Diderot et d'Alembert qui en assure la célébrité (Marguin 1994 : 46-62). Plus encore que Descartes et sa machine à polir les lentilles, Pascal a conçu les éléments mécaniques de son projet technique avec rigueur. Pour la réalisation concrète, il en confie cependant la tâche à des horlogers et c'est alors que les ennuis commencent. Cette fois, ce ne sont pas tant les contraintes matérielles qui sont en cause que le cahier des charges.

Pascal confie au chancelier Séguier, dans sa lettre dédicatoire de 1645, au moment où sont produits les premiers modèles de sa machine à calculer, quelle fut sa démarche et sa relation de dépendance à l'égard des artisans (lui-même «n'ayant pas l'industrie de 
manier le métal et le marteau comme la plume et le compas ») qui induisent cependant la subsidiarité de leurs compétences :

«Les lumières de la géométrie, de la physique et de la mécanique m'en fournirent le dessein, et m'assurèrent que l'usage en serait infaillible si quelque ouvrier pouvait former l'instrument dont j'avais imaginé le modèle. Mais ce fut sur ce point que je recontray des obstacles aussi grands que ceux que je voulois éviter, et auxquels je cherchois un remède ${ }^{9}$."

13 Pascal a donc imaginé les rouages et le modèle mécanique qui permet d'effectuer les opérations voulues, y compris l'indispensable sautoir. Les horlogers auraient ensuite dû avoir toute liberté pour agencer ces pièces de manière à rendre la machine solide et performante pour un prix raisonnable; mais Pascal ajoute une exigence qui change la donne et compromet sa constructibilité. Il réfléchit au problème de l'erreur de calcul : le mécanisme ne pouvant se tromper, les erreurs ne peuvent être qu'humaines. Il faut donc concevoir une machine qui soit facile d'utilisation pour les éviter. La pascaline affiche sur deux lignes placées l'une en dessous de l'autre additions et soustractions ; en fonction du résultat que l'opérateur veut lire, une petite baguette vient masquer le résultat non souhaité. Cette contrainte de lisibilité n'a l'air de rien mais elle signifie concrètement, pour les horlogers, que deux mouvements perpendiculaires devaient être ramenés sur le même plan pour être visibles l'un en dessous de l'autre. Cela complique le mécanisme et cette complication augmente le coût et la fragilité de l'appareil. Un autre problème concerne le sautoir: agissant par gravité, il est extrêmement sensible aux cahots, ce qui nécessite pour que la machine fonctionne bien qu'elle soit posée sur une table et non secouée. Or, Pascal aurait souhaité, pour séduire son public de trésoriers toujours pressés d'aller recouvrer leurs fonds, que la pascaline puisse être utilisée dans un carrosse, à tout le moins en ambulatoire. Tout ceci conduit à l'échec commercial de la première machine à calculer: après une cinquantaine de prototypes réalisés entre 1642 et 1645 et malgré un privilège royal accordé en 1649 , il ne s'en vend pas plus d'une vingtaine. La pascaline va rejoindre dans les cabinets de curiosités ces autres objets que l'on admire d'autant plus qu'ils sont rares et pour tout dire inutiles.

14 L'exigence ergonomique, à laquelle on associe aussi certaines recherches de Léonard, ne nous semble pas aujourd'hui relever de l'excentricité. On peut même être tenté de voir en Pascal un précurseur de ces ingénieurs modernes, en particulier dans l'informatique, qui conçoivent leurs objets techniques en fonction de leur facilité d'usage (Alizart 2017). Mais, comme Descartes, Pascal raisonne abstraitement, sans tenir compte des critères de faisabilité, ce qui aboutit à des contradictions: soit la machine est solide et bon marché mais elle n'est pas pratique, soit elle est pratique mais fragile et chère. Pascal ne l'ignore point puisqu'il possède chez lui un modèle toutterrain et non ergonomique qu'il emploie pour son propre compte, mais il choisit de passer outre, persuadé que le risque d'erreur humaine est ce qu'il faut éviter à tout prix. 


\section{Desargues et la taille des pierres}

15 Girard Desargues (1591-1661), est l'un des fondateurs de la géométrie dite projective. À ce titre, ses travaux jouent un rôle fondamental dans l'élaboration de la culture technique contemporaine. Son œuvre est cependant plus importante que sa personne n'est connue. Il est ainsi le premier à proposer une théorie unifiée des coniques (deux théorèmes portent toujours son nom), bien que quelques jaloux l'accusent d'avoir plagié Pappus et Apollonius. Issu d'une famille de juristes et de négociants lyonnais, il rencontre à Paris, Mersenne et Descartes, puis publie son premier traité en 1639 (celui sur les coniques) qui inspire le jeune Pascal. Quelques temps plus tard, il ouvre un cours de perspective destiné aux tailleurs de pierre, graveurs et autres charpentiers qui voudraient enrichir leur pratique d'un savoir théorique. Ses élèves ne sont pas des anonymes : le menuisier Huret, le graveur Bosse, le peintre La Hire, le maçon Hureau ou l'appareilleur Bressy. De ce cours, il tire un second ouvrage : le Brouillon-projet pour la taille des pierres, publié en août $1640^{10}$.

16 Cet opuscule constitué de quatre grandes pages in-folio et de cinq planches de dessins fait aussitôt polémique (Taton 1951 : 38-58). Il faut dire que Desargues y adopte un ton assez dur envers ceux qui se contentent d'une "pratique tâtonneuse » à laquelle il oppose le savoir des "purs géomètres ", seuls capables de juger de l'exactitude des méthodes générales de construction. S'ensuit une série de pamphlets acides sur fond d'accusation réciproque de plagiat qui implique aussi des libraires. Les ennemis de Desargues soulignent sa prétention démesurée et son incompétence pratique, rappelant cruellement que sa seule réalisation alors connue est... un escalier tenu par lui pour un chef-d'œuvre! À partir de 1644, la dispute s'envenime avec la publication d'un libelle hostile à Desargues écrit par un maître-maçon parisien nommé Curabelle. Desargues le menace d'un procès devant le Parlement au nom d'un privilège qu'il a obtenu du roi en 1642 pour protéger ses œuvres et se dit victime d'une «caballe d'ouvriers » :

\footnotetext{
«Moy je ne suis artisan de la main d'aucune sorte d'Art; je n'ay que la simple connoissance de la raison de l'effect des règles du peu de traicts dont je propose la pratique; et je n'ay point une caballe d'ouvriers comme il [Curabelle] a ses compagnons cotteries; par le nombre et par la crierie desquels il voudroit m'opprimer; et comme il sçait que je ne travaille point de la main, il me voudroit engager à quelque travail effectif de maçonnerie ou bien à dépendre de la discrétion des ouvriers, ses compagnons cotteries; ce qui ne seroit ny juste ny raisonnable. $»^{11}$
}

Malgré ces rodomontades, Desargues finit par être blessé par les accusations d'incompétence technique. À la fin des années 1640, il abandonne peu à peu ses recherches théoriques et entame, comme pour prouver à ses adversaires qu'ils ont tort de le prendre pour un simple plumitif, une carrière d'architecte qui le fait travailler sur des chantiers d'hôtels particuliers parisiens et lyonnais ${ }^{12}$.

Cette fois, le savant a dû faire face à plus qu'une incompatibilité de cultures; il s'est heurté à un mur, celui des jurandes qui n'entendent pas se faire donner la leçon par un bel esprit sorti du néant à si bon compte ${ }^{13}$. Faiblement soutenu par ses pairs, doté d'un caractère ombrageux, il a cédé. Sa méthode de projection pourtant lui survit et, par 
l'intermédiaire de son élève doué, le graveur Abraham Bosse, elle prospère par la suite en Hollande, et influença les travaux de Monge, inventeur de la géométrie descriptive si déterminante pour l'émergence d'une culture technoscientifique unifiée. Car ce qui est en jeu, c'est la nécessaire articulation entre conception et réalisation, articulation qui cause encore ici tant de malentendus. On doit à la révolution industrielle, sans doute pour des raisons plus économiques qu'épistémiques, d'avoir contribué à résoudre ce conflit latent: c'est le client qui va pousser savants et artisans à se mettre d'accord pour mieux collaborer, comme l'illustre l'exemple bien connu de la machine à vapeur (Hilaire-Pérez, 2003).

\section{Conclusion}

Notons que les exemples qui ont été traités ci-dessus concernent les ennuis rencontrés par quelques savants avec quelques artisans bien davantage qu'avec quelques ingénieurs. Outre que la définition de l'«ingénieur " varie au cours de la période considérée, on aurait tort néanmoins de n'y voir que la défense d'intérêts corporatistes de simples faiseurs opposés aux "progrès » inéluctables des connaissances produites par de rares génies. Ces cultures sont poreuses; elles sont appelées à un nécessaire dialogue et se complètent pour définir notre modernité même, ainsi que Paul Valery l'a magnifiquement exprimé à propos de l'esprit vagabond de Léonard :

\footnotetext{
«Esprit de finesse, esprit de géométrie, on les épouse, on les abandonne, comme fait le cheval accompli ses rythmes successifs... Il doit suffire à l'être suprêmement coordonné de se prescrire certaines modifications cachées et très simples au regard de la volonté, et immédiatement il passe de l'ordre des transformations purement formelles et des actes symboliques au régime de la connaissance imparfaite et des réalités spontanées ${ }^{14}$.» (Valéry 1919)
}

20 pas mais sait aussi lui imposer le galop et le franchissement d'obstacles. C'est cette souplesse qui a manqué à Descartes, Pascal et Desargues ; si bien qu'on peut se demander si ce ne sont pas ces derniers qui défendent des intérêts corporatistes (ceux des mathématiciens en mal de reconnaissance et de professionnalisation ${ }^{15}$ ) face à des artisans et des ingénieurs mieux au fait des réalités et des attentes de leur temps ${ }^{16}$. Le mépris, bien inscrit dans la culture occidentale, entre les "penseurs» et les "faiseurs ", atteint ici son paroxysme au moment même où leur collaboration est plus que jamais nécessaire. Chronologiquement, les trois exemples dont il a été question se situent avant la fondation des grandes académies royales, dont il semble qu'elles aient pu jouer un rôle plus important qu'on ne l'a dit dans le rapprochement et le dialogue entre les deux cultures (Delaunay 2017). Au temps de Léonard, le chemin qui mène de la bibliothèque à l'atelier est encore court. Au temps de Descartes, Pascal et Desargues, il s'est allongé et se trouve semé d'obstacles. Il est pourtant plus fréquenté que jamais et c'est cette « intellectualisation de la pensée sur la matière » (Garçon $2007: 26)$ propre à la période galiléenne qui crée les tensions que le siècle des Lumières s'acharne à résoudre.

Ainsi, est-il tout aussi vain de s'extasier devant les belles machines de Léonard (qui ne sont ni si originales ni si performantes) que de pointer du doigt leur " irréalité »: 
Léonard a cette chance, avec quelques autres et parce qu'il a vécu à une époque qui le permettait encore, de pouvoir considérer tour à tour les deux points de vue (celui du savant qui imagine et celui de l'artisan ou de l'ingénieur qui fabrique) (Lefèvre 2004). Se faisant, il nous révèle, non sans nous troubler, cette sempiternelle hésitation qui constitue l'un des moteurs des grands bouleversements scientifiques et techniques qui eurent lieu entre Renaissance et Lumières.

\section{BIBLIOGRAPHIE}

ALIZART Mark, Informatique céleste, Paris, PUF, 2017

BRIOIST Pascal, Les audaces de Léonard de Vinci, Paris, Stock, 2019

BURNETT Graham D., Descartes and the Hyperbolic Quest: Lens Making Machines and their Significance in the Seventeenth Century, Philadelphie, American Philosophical Society, 2005

DELAUNAY Bernard, L'Académie royale des Sciences et la technique, XVII ${ }^{e}$-XVIII ${ }^{e}$ siècle, Paris, $\mathrm{H}$. Champion, 2017

GARÇON Anne-Françoise, « Science et Technique, Technique et Science... Histoire d'une complémentarité historiquement occultée », Atala, 10, 2007, p.15-28

GARÇON Anne Françoise, « Une brève histoire de la culture technique européenne et de sa relation à l'innovation », ISTE OpenScience [En ligne], vol.17, n², 2017, DOI : 10.21494/ISTE.OP. 2017.0156

GOLDSTEIN Catherine, «L'honneur de l'esprit : de la République des mathématiques », dans COSANDEY Fanny (dir.), Dire et vivre l'ordre social en France sous l'Ancien Régime, Paris, Éd. de l'EHESS, 2005, p.191-230

HILAIRE-PÉREZ Liliane, « Pratiques inventives, cheminements innovants, crédits et légitimations ", dans HILAIRE-PÉREZ Liliane et GARÇON Anne-Françoise (dir.), Les chemins de la nouveauté : innover, inventer au regard de l'histoire, Paris, Éd. du CTHS, 2003, p.9-38

KOYRÉ Alexandre, « Léonard de Vinci, 500 ans après ", dans ID., Études d'histoire de la pensée scientifique, Paris, PUF, 1966, p. 99-116

LEFÈVRE Wolfgang (dir.), Picturing machines 1400-1700, Cambridge (Mass.), MIT Press, 2004

MARGUIN Jean, Histoire des instruments et machines à calculer. Trois siècles de mécanique pensante, 1642-1942, Paris, Hermann, 1994

MICHAUX Bernard, «Pascal, Descartes et les artisans », dans Les Pascal à Rouen : 1640-1648. Colloque de l'Université de Rouen... 17, 18, 19 novembre 1999, Rouen, PUR, 2001, p.197-215

ORTOLI Sven et WITKOWSKI Nicolas, La Baignoire d'Archimède : petite mythologie de la science, Paris, Seuil, 1996

PÉROUSE DE MONTCLOS Jean-Marie, L'architecture à la française du milieu du XV ${ }^{e}$ siècle à la fin du $\mathrm{XVIII}^{e}$ siècle, Livre 2. La stéréotomie pierre de touche de la manière française, Paris, Éditions A. et J. Picard, 2001 
RAYNAUD Dominique, Sociologie des controverses scientifiques, Paris, Éd. Matériologiques, 2018

RONNA Antoine, Léonard de Vinci, peintre-ingénieur-hydraulicien (1452-1519), Paris, Ph. Renouard, 1902

ROSSI Paolo, Les philosophes et les machines, 1400-1700, Paris, PUF, 1996

SAKAROVITCH Joël, Épures d'architecture. De la coupe des pierres à la géométrie descriptive XVI ${ }^{e}$-XIX siècles, Bâle, Birkhäuser Verlag AG. Collection Science Networks, 1998

SAKAROVITCH Joël, « Le fascicule de coupe des pierres de Girard Desargues », dans Encyclopédie des métiers : la maçonnerie et la taille de pierre, Paris, Presses du Compagnonnage, 2010, p. 121-147

SHAPIN Steven, La révolution scientifique, Paris, Flammarion, 1998

STENGERS Isabelle, «Les affaires Galilée », dans SERRES Michel (dir.), Éléments d'histoire des sciences, Paris, Bordas, 2003, p.337-375

TATON René, L'Euvre mathématique de Desargues, Paris, PUF, 1951

VALÉRY Paul, Introduction à la méthode de Léonard de Vinci, Paris, Éd. de la NRF, 1919.

VECCE Carlo, Léonard de Vinci, Paris, Flammarion, 2001

VÉRIN Hélène, La gloire des ingénieurs. L'intelligence technique du XVI au XVIII ${ }^{e}$ siècle, Paris, Albin Michel, 1993

\section{NOTES}

1. Au sujet des mythes de la culture scientifique et technique, voir (Ortoli, Witkowski 1996).

2. VASARI Giorgio, La terza et ultima parte delle Vite degli architetori pittori et scultori, Florence, L. Torrentino, 1550, p.562.

3. A. Koyré s'est amusé de cette image d'« homme à tout faire » construite par l'historiographie positiviste qui n'hésita pas à proclamer Léonard "plus grand esprit moderne, fondateur des techniques et de la science modernes, précurseur de Copernic, de Vésale, de Bacon et de Galilée » (Koyré 1966 : 100-102).

4. DESCARTES René, Discours de la methode : pour bien conduire sa raison, \& chercher la verité dans les sciences : Plus La Dioptrique, et Les Meteores, Paris, Th. Girard, 1637, p.137-153.

5. Notons que Léonard, dans un fameux dessin du Codex Atlanticus (fol. 1103), a lui aussi conçu une machine à polir les lentilles mais non pour résoudre le problème de l'aberration qui n'avait pas encore été constaté à son époque.

6. Euvres de Descartes, éd. ADAM Charles et TANNERY Paul, Correspondance, t.I, Paris, Vrin, 1987, lettre de Ferrier à Descartes du 26 octobre 1629, p.46.

7. Dans une lettre du 31 mars 1638 à Mersenne, Descartes exprime sa déception à l'égard de l'artisan parisien : « Pour Ferrier, laissez-le faire ; il y a grande apparence qu'il n'achèvera rien, et je croy que le moindre petit tourneur ou serrurier seroit plus capable que luy de voir l'effect des lunetes" (ibid., t. II, Paris, Vrin, 1988, p. 85). Un an plus tard, dans une lettre au même du 9 février 1639, apprenant qu'il se fabrique à Naples des lentilles hyperboliques, Descartes accuse Ferrier d'avoir trahi son procédé. Ironie de cette affaire, le grand mathématicien fait ici preuve d'une jalousie et d'une paranoïa de boutiquier que l'on attribue d'ordinaire plus volontiers aux artisans de son temps (ibid., p.493).

8. DESCARTES René, op. cit., p. 153. Souligné par nous. 
9. Lettre dédicatoire à Monseigneur le Chancelier sur le sujet de la machine nouvellement inventée par le Sieur B. P. pour faire toutes sortes d'opérations d'arithmétique, par un mouvement réglé, sans plume ni jetons, s. 1., 1645, p.3.

10. DESARGUES Girard, Brouillon-project d'exemple d'une manière universelle touchant la practique du trait à preuves pour la coupe des pierres en l'architecture, Paris, 1640.

11. DESARGUES Girard, Discours au vrai de ce qui a esté la cause de faire cet escrit, Paris, 1644, p.24.

12. Cette querelle a fait l'objet d'une récente analyse de controverse (Raynaud $2018: 245-272$ ) qui complète et confirme par des documents inédits les interprétations de R. Taton.

13. «Si Desargues a géométriquement raison, il a pratiquement tort» (Sakarovitch 2010 : 125). Le Brouillon-Project, aujourd'hui quasiment introuvable, est reproduit et transcrit dans ce même article, p. 131-147. Un siècle plus tard, chez La Rue (1728) ou Frazier (1737), théorie et pratique sont réconciliées et la stéréotomie enfin enseignée aux architectes (Sakarovitch 1998 ; Pérouse de Montclos 2010).

14. Préface à la nouvelle édition. La première édition de l'Introduction à la méthode de Léonard de Vinci date de 1895.

15. La "crise » sociale subie par la profession des mathématiciens au milieu du XVII ${ }^{\mathrm{e}}$ siècle (Goldstein 2005) n'est sans doute pas sans lien avec la baisse de prestige de l'astrologie qui les avait durant des siècles rendus indispensables aux princes, tandis que l'usage politique des mathématiques (par les statistiques notamment) n'est pas encore développé.

16. Par un touchant aveu d'imprécision, l'ingénieur militaire Jean Errard balaye d'un revers de main toutes les jérémiades des géomètres qui se sont émus avant lui de la non-constructibilité à la règle et au compas de l'heptagone, forme qu'il propose néanmoins comme modèle de construction dans sa Fortification démonstrée et réduicte en art (1600) : "Je ne chercheray autre supputation plus exacte, puisque les parties défaillantes de celle-cy sont insensibles ». Belle entorse à la norme d'exactitude, du moment que la muraille dévie correctement les boulets.

\section{RÉSUMÉS}

Il existe un mythe Léonard. Forgé dès sa mort, il réunit l'artiste habile à l'ingénieur à l'imagination débordante. On lui prête sans doute trop : tantôt érudit, tantôt homme sans lettres, tantôt visionnaire, tantôt plagiaire. L'ambiguïté même de sa figure, réelle ou construite, révèle un malentendu récurrent entre culture savante, culture technique et savoir-faire artisanal durant l'époque moderne. L'article examine quelques cas célèbres de ces grandes incompréhensions du XVIIe siècle, considérées en tant que modèles d'une science de l'invention encore à ses balbutiements et qui ne prendra sa forme actuelle qu'à l'ère industrielle.

There is a Leonardo's myth. Framed after his death, this myth combines the virtuoso artist with the imaginative ingegnere. No doubt, we attribute too much to his talent: man without instruction or scholar, forward-thinking or plagiarist. As an actual image or a constructed one by history, the figure of Leonardo exhibits a constant misinterpretation between scientific, technical and craft cultures. This paper looks at some examples of those misunderstandings of the seventeenth century, as if they were models of a new science of invention, which is going to take its contemporary form only at the industrial area. 
INDEX

Mots-clés : histoire des techniques, histoire des sciences, invention, culture technique, machine Keywords : history of technology, history of science, invention, machine, technical culture

\section{AUTEUR}

\section{LAURENT-HENRI VIGNAUD}

Né en 1971, maître de conférences à l'université de Bourgogne, Laurent-Henri Vignaud est historien des sciences, spécialiste du savoir naturaliste à la Renaissance. Il a publié en 2016 un manuel universitaire consacré à l'histoire des sciences et des techniques de la Renaissance aux Lumières. 